\title{
Babylonian observations of a unique planetary configuration
}

\section{Teije de Jong ${ }^{1}\left[\right.$ [D Hermann Hunger ${ }^{2}$}

Received: 28 May 2020 / Published online: 3 August 2020

(c) The Author(s) 2020

\begin{abstract}
In this paper, we discuss Babylonian observations of a "massing of the planets" reported in two Astronomical Diaries, BM 32562 and BM 46051. This extremely rare astronomical phenomenon was observed in Babylon between 20 and 30 March 185 BC shortly before sunrise when all five planets were simultaneously visible for about 10 to $15 \mathrm{~min}$ close to the horizon in the eastern morning sky. These two observational texts are not only interesting as records of an extremely rare planetary configuration, but also because (1) the observers appear to be confused by the presence of all planets simultaneously and mix them up in their reports, and (2) the two reports of the same observations are so different that we are forced to conclude that they were carried out by two different observers. There is an additional astronomical event which makes this planetary configuration even more unique: the exact conjunction of the planets Mars and Jupiter in the afternoon of 25 March 185 BC. An exact conjunction, where two planets are so close together that they appear as one object in the sky, is also extremely rare. Although this exact conjunction between Mars and Jupiter occurred during the day so that it was not observable, it was correctly predicted by the Babylonian scholars: a remarkable achievement and a nice illustration of their astronomical craftsmanship. Finally, our study clearly exposes one of the limitations of Babylonian naked-eye astronomy. When first appearances of the planets Mercury, Mars and Saturn are expected around the same date, it is nearly impossible to correctly identify them because their expected positions are only approximately known while they have about the same visual magnitude so that they become visible at about the same altitude above the horizon.
\end{abstract}

Communicated by Alexander Jones.

Teije de Jong

t.dejong@uva.nl

Hermann Hunger

hungerh5@univie.ac.at

1 Astronomical Institute Anton Pannekoek, University of Amsterdam, Amsterdam, The Netherlands

2 Institut für Orientalistik, Universität Wien, Vienna, Austria 


\section{Introduction}

At present, one of us $(\mathrm{HH})$ is preparing the edition of volume IV of the Astronomical Diaries and Related Texts from Babylonia (Hunger 2021). This final volume will present transliterations and translations of texts preserved on (fragments of) tablets that were not included in the first three volumes of the Astronomical Diaries (Sachs and Hunger 1988, 1989, 1996) because their contents are so poorly preserved and so fragmentary that they could not be easily dated. During the preparation of this final volume, it turned out that the astronomical information preserved on some of these scraps and fragments was occasionally still sufficient to come up with a reliable dating of the observations. In cases where the text seemed to contain sufficient astronomical information but where the dating process required more advanced astronomical techniques, the other one of us ( $\mathrm{TdJ}$ ) was occasionally called in for assistance.

Here, we report on one of these texts, BM 32562 which, once dated, turned out to contain observations of a unique planetary configuration, a so-called massing of the planets, when all five visible planets can be simultaneously observed while being very close together. This extremely rare astronomical phenomenon was observable in Babylon between 20 and 30 March 185 BC shortly before sunrise when all five planets were simultaneously visible for about 10-15 min close to the horizon in the eastern morning sky.

The observational text BM 32562 is not only interesting in itself as the record of an extremely rare planetary configuration, but also because, (1) the observer appears to be confused by the presence of all planets simultaneously and mixes them up in his report, (2) the observation is a parallel of the one reported in BM 46051, published earlier as part of Astronomical Diary No. -185 (Sachs and Hunger 1989, 351-353), where the observer is also, but differently, confused, (3) the two reports of the same observations are so different that we are forced to conclude that they were carried out by two different observers.

\section{Tablet BM 32562}

The tablet BM 32562 is a lower left corner part and measures 36 by $46 \mathrm{~mm}$. Of its upper part not much seems to be missing. It was originally registered by the British Museum as $\mathrm{S} \uparrow 76-11-17,2305$. It is part of a collection that was bought from an antiquities dealer. The provenance is most likely Babylon, but nothing can be said about the archaeological context. A transliteration of the text and photographs of the tablet will be published by Hunger (2021).

BM 32562

'Obv.

$\left.\# 1^{\prime} \quad \ldots . . . ..\right]$

$\# 2^{\prime} \quad 1 / 2$ cubit $[\ldots .$.$] ; all ni [$ ght.... $]$ first appearance $[\ldots .$.$] it stood [\ldots$. behind.... $]$

\#3' to the east; it was bright (and) high, [time interval (to sunrise/sunset)]...., 
\#4' (ideal) first appearance on the 26th. The 30th, Saturn's first appearance in.... [....]

$\# 5^{\prime} \quad$ it stood [....] in front of Mercury to the west, $1 / 2$ cubit below.... [...., ]

$\# 6^{\prime}$ it stood [....] behind Mars to the east.... [....]

$\# 7^{\prime} \quad$ (ideal) first appearance on the 29th. That month, [....]

Left edge

$\# 1 \quad[\ldots .]$.1 cubit, the moon being.... [....]

$\# 2 \quad \ldots$. Night of the 4 th, the moon was $[\ldots .$.$] above \zeta$ Tauri $[\ldots .$.

Rev.'

\#1 [Night] of the 5th, the moon was [....] above [....]

\#2 2? cubits, the moon [having passed] a little to the east [....]

\#3 Venus? was 8 fingers above Mars; last part of the night,.... [....]

\#4 [.... it was se]t? toward its inside [....]

\#5 ...., rain shower

(blank)

\#6 That? month?, [....].... [....]

\section{Commentary:}

This short Diary covers only a limited number of days. For the second month, it goes only to day 5. Of the first month, only a few days were recorded. It is hard to say when the Diary started but probably not earlier than day 22. It may be that the unusual planet configuration during these days was the reason to collect the observations on a special tablet. Other short Diaries do exist, but they start with the beginning of a month and do not start a new month when the preceding one is incomplete.

\section{Dating of BM 32562}

In attempting to date the planetary observations recorded on BM 32562, we tried several different approaches. Rather than reporting on our trial and error process, we describe here the two steps of the final successful dating.

Lines 4-6 on the obverse side of the tablet state that the planets Mercury, Mars and Saturn were close together shortly before sunrise on the 30th day of the month. So we first searched for all dates between $600 \mathrm{BC}$ and $0 \mathrm{AD}$ when: (1) each of these three planets was within $10^{\circ}$ of the two other ones, and (2) the day number in the Babylonian lunar calendar was 29,30 or 1 . The choice of $10^{\circ}$ is based on the fact that in the Astronomical Diaries distances between two planets and between planets and Normal Stars are usually only specified if they are less than about 5 cubits, and limiting the search to the 29th, 30th or 1st day of the lunar month is due to the uncertainty in the observation of the first visibility of the lunar crescent which creates an uncertainty margin of \pm 1 day. This search resulted in nine sets of candidate Julian dates for the 30th day in the text: 5-7 June 591 BC, 13-15 June 410 BC, 
11-12 July 380 BC, 19-20 July 378 BC, 19-20 March 185 BC, 27 March 183 BC, 2-4 July 173 BC, 23-24 April 153 BC and 26-28 January 12 BC.

Of these nine sets of candidate dates, 20 March $185 \mathrm{BC}$ is the only one for which a conjunction of the Moon with the Normal Star $\zeta$ Tauri occurs 4 days later during the night of the 4th day of the next month recorded in line 2 on the left edge of tablet BM 32562. ${ }^{1}$ This date is further confirmed by the passing of the Moon by another Normal Star during the following night (of the 5th day) referred to in lines 1 and 2 on the reverse side of the tablet. ${ }^{2}$

Using the Babylonian calendar calibration of Parker and Dubberstein (1956), we find that the 2 months for which observational records are preserved on BM 32562 are month XII and $\mathrm{XII}_{2}$ of year 126 of the Seleucid Era. Parker and Dubberstein, using the lunar crescent visibility criterion formulated by Carl Schoch (Langdon et al. 1928), computed a month length of month XII of 29 days while the observational record shows that in actual fact month XII of SE 126 counted 30 days. This difference is not surprising because it was shown by Huber et al. $(1982,27)$ that the crescent visibility criterion used by Parker and Dubberstein leads to differences between computed and actually observed Babylonian month lengths in about 7\% of the cases. Since apparently the lunar crescent was not sighted in the evening of 20 March 185 BC, sunset on that day marked the beginning of day 30 of month XII of SE 126 rather than day 1 of the next month $\mathrm{XII}_{2}$.

After having dated the planetary observations recorded on BM 32562, we found that tablet BM 46051, published earlier as exemplar B of Diary No. -185 (Sachs and Hunger 1989), contained observations of the same unique planetary configuration. Sachs and Hunger dated this tablet on the basis of the planetary observations in the text. Their dating is confirmed by the observations of the passing of the Moon by seven Normal Stars recorded in lines 3'-9' on the reverse of BM 46051. To be able to compare the two observational reports of the same astronomical phenomena, we render here Hunger's translation of the obverse and of the first two lines of the reverse of BM 46051 (Sachs and Hunger 1989, 351-352).

BM 46051

'Obv.'

$\# 1^{\prime} \quad$ [.... ar]ound the 30th, Saturn's first appearance be[low Mars, ]

\#2' [it stood 2 cubits in fro]nt of Jupiter to the west, $3 \frac{1 / 2}{2}$ cubits behind Mercury to the east; rising of Saturn to sunrise: $16^{\circ}$;

\#3' (ideal) first appearance [on the xth]. That month, the equivalent was: barley, 2 pān 2 sūt; dates, 4 pān 3 sūt; mustard,

\#4' [....]....; cress, 1 pān 2 sūt; sesame, 4 sūt; wool, 4 minas

\#5' were sold for 1 shekel of silver. At that time, around the 22nd, Jupiter's first appearance, it stood 2 cubits behind Saturn

\#6' [to the east;] rising of Jupiter to sunrise: $10^{\circ}$; (ideal) first appearance on the 21 st; the 22nd, Saturn's first appearance in the beginning of Pisces

\footnotetext{
1 At $19: 30 \mathrm{~h}$ on 23 March $185 \mathrm{BC}$, the Moon was $2.4^{\circ}$ above and $2.0^{\circ}$ behind the star $\zeta$ Tauri.

2 At $19: 30 \mathrm{~h}$ on 24 March $185 \mathrm{BC}$, the Moon was $6.0^{\circ}$ above and $0.4^{\circ}$ to the east of the star $\gamma$ Geminorum.
} 
\#7' [2 cubits in] front of Jupiter to the west; rising of Saturn to sunrise: $14^{\circ}$; (ideal) first appearance on the 21st; Venus

\#8' [....] reached Aquarius; around the 30th, Saturn's first appearance 1/2 cubit below Mars, it stood 2 cubits

\#9' [in front of Jupiter to] the west, $3 \frac{1}{2} 2$ cubits behind Mercury to the east; rising of Saturn to sunrise: $16^{\circ}$ ?;

$\# 10^{\prime} \quad[\ldots]$

\section{'Rev.'}

\#1' $[\ldots .$.$] having passed [\ldots .$.$] to the east. Night of the 5$ th, the $m[$ oon was....] 8 fingers below? $\gamma$ Geminorum;

\#2' last part of the night, Mars was 3 fingers in front of Jupiter,

\#3' [...] The 5th, in the morning, very overcast, rain shower. Night of the 6th, the moon was in front of

Notice that line 1 of the text on the obverse of BM 46051 confirms that month XII of SE 126 indeed counted 30 days. In the next sections, we analyse the planetary observations recorded on tablets BM 32562 and BM 46051 combined, in chronological order.

\section{The planetary observations recorded on BM 32562 and BM 46051}

In line with Babylonian astronomical practice, we first predict which planets were visible at the start of month XII of SE 126 and what first appearances of planets were expected to occur during that month. We know that these kind of predictions were routinely made by the Babylonian astronomers and that they were written up in so-called Almanacs (Hunger 2014). The predictions were made based on previous observations one or more so-called Goal-Year periods back. To understand the way in which Goal-Year periods were used to predict planetary phenomena, we refer the reader to Steele (2011). Dates on which first appearances were expected based on such predictions are often referred to in the Diaries. In the two Diaries investigated here, we find references to "ideal first appearances" of Jupiter and Mercury on the 21st day of month XII, of Mars on the 26th and of Saturn on the 29th day.

Using the astronomical techniques briefly described in de Jong (2012), we computed dates of first and last appearances of the planets in Babylon in 185 BC adopting a local atmosphere characterized by a visual extinction of 0.27 magnitudes per air mass. ${ }^{3}$ This resulted in the following planetary forecast for month XII of SE 126. At the beginning of that month Venus was visible as morning star at the end of Capricorn, and first appearances were expected of Mercury on the 21st, of Jupiter on the $23 \mathrm{rd}$, of Saturn on the 27 th and of Mars on the 30th day of the month. These

\footnotetext{
3 In earlier studies of ancient Babylonian observations of stars and planets, de Jong (2002, 2007, 2012) found average atmospheric extinction values varying from $0.25 \pm 0.05$ magnitudes per airmass for Babylon to $0.34 \pm 0.07$ for Uruk. Due to variable weather conditions, the actual atmospheric extinction in Babylon may vary from day to day between about 0.15 and 0.50 magnitudes per air mass. For the sake of brevity values of the atmospheric extinction will often be given without its units of magnitudes per airmass.
} 
predicted dates may vary by up to \pm 3 days due to variable weather conditions and are therefore fully consistent with the Babylonian expected "ideal" dates of first appearance in the text.

One might expect that this close coincidence of the dates of first appearances of four planets would have aroused some excitement among the scholarly astronomical community in Babylon, although none of this is reflected in the dry professional jargon of the observational records in the Diaries. The period that all five planets could be observed simultaneously in the early morning shortly before sunrise ended on the 10th day of month $\mathrm{XII}_{2}$ with the last appearance of Mercury.

We start our analysis of the Babylonian observations in months XII and $\mathrm{XII}_{2}$ of SE 126 with lines 7 and 8 on the observe of BM 46051. These lines are part of the observational summary of month XII. Such summaries are found at the end of the month in almost all Astronomical Diaries. Lines 7 and 8 contain the following record of the planet Venus reaching the zodiacal sign of Aquarius:

BM 46051, 'Obv.'

$\# 7^{\prime} \quad$.....; Venus

$\# 8^{\prime} \quad[\ldots .$.$] reached Aquarius;$

The Babylonian criterion for a planet to reach the sign of Aquarius was its passage of the Normal Star $\delta$ Capricorni, which has a longitude in the Babylonian zodiac of $300^{\circ}$ (Huber 1958; Britton 2010). This happened in the late night of 25 February 185 BC, i.e. day 6 of month XII of SE 126.

The next observation in chronological order is found in lines 5-7 on the obverse of BM 46051 where first appearances of Jupiter and Saturn are reported, again as part of the observational summary at the end of month XII:

BM 46051, 'Obv.'

\#5' ... At that time, around the 22nd, Jupiter's first appearance, it stood 2 cubits behind Saturn

\#6' [to the east;] rising of Jupiter to sunrise: $10^{\circ}$; (ideal) first appearance on the 21 st; the 22nd, Saturn's first appearance in the beginning of Pisces

\#7' [2 cubits in] front of Jupiter to the west; rising of Saturn to sunrise: $14^{\circ}$; (ideal) first appearance on the $21 \mathrm{st} ; \ldots$

The text reports that around the 22nd day of month XII of SE 126 in the early morning, shortly before sunrise, the planets Jupiter and Saturn both appeared for the first time (after having been invisible for about 40 and 50 days, respectively). According to the text, both planets were expected to have their ("ideal") first appearances on day 21 . The original observations for day 22 of month XII are not preserved on the tablet. 


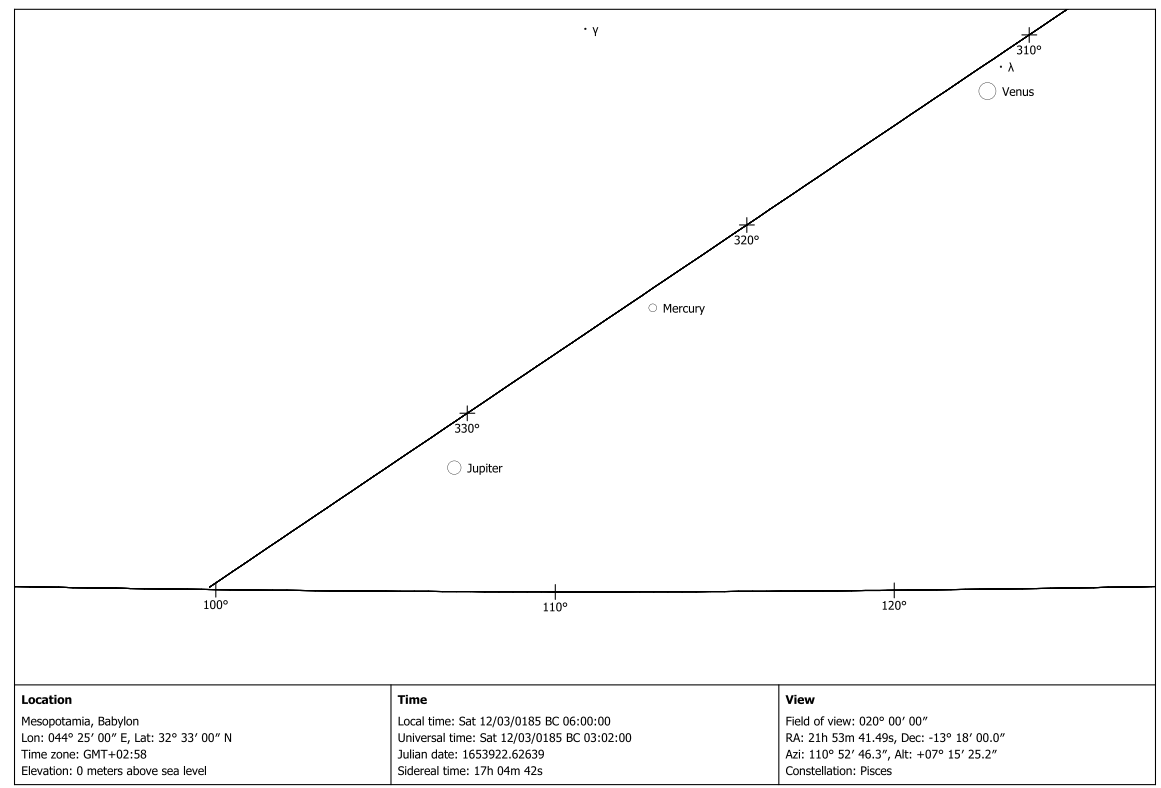

Fig. 1 The eastern morning sky in Babylon on day 22 of month XII in SE 126 (12 March 185 BC) at 6:00 h Local Time. The planets Jupiter, Mercury and Venus are visible. The sizes of the circles representing the planets are a measure of their relative brightness. The horizon and the ecliptic are indicated with azimuth and ecliptic longitude marked at $10^{\circ}$ intervals. [Produced with Cybersky 5.1.2; copyright $\odot$ 2018 Stephen M. Schimpf]

Obviously, the scribe here makes the mistake of writing Saturn instead of Mercury because Saturn's first appearance is expected more than one week later. ${ }^{4}$ Indeed, in the next line (\#8) of the text he reports the first appearance of Saturn on the 30th day of the month. The situation is illustrated in Fig. 1 where we show the positions of Jupiter, Mercury and Venus near the eastern horizon shortly before sunrise at 5:50 h local time on the $22 \mathrm{nd}$ of month XII.

Contrary to what is reported in the text, Saturn (Mercury!) was in reality about 3 cubits $\left(\sim 7^{\circ}\right)$ behind Jupiter rather than 2 cubits $\left(\sim 4^{\circ}\right)$. The inaccuracy of this estimate can be understood if one takes into account that Jupiter and Mercury were not simultaneously visible in the morning of the 22 nd. Mercury was visible from 5:31 to $5: 48 \mathrm{~h}$, rising from altitude $2.9^{\circ}$ to $6.2^{\circ}$, and Jupiter from 5:52 to 5:59 h, rising from $2.3^{\circ}$ to $3.7^{\circ} .^{5}$ Since in this case the distance in ecliptic longitude between the two planets could not be directly measured in the sky, the difference in longitude may have been estimated from the difference in time between the visibility of Mercury

\footnotetext{
${ }^{4}$ See the date on which the first appearance of Saturn is expected based on modern computation quoted in the first paragraph of this section and the Babylonian "ideal" expected date in line 7 on the obverse of BM 32562.

5 These visibility windows and altitude ranges are computed for an atmospheric extinction of $k=0.25$ magnitudes per airmass. For extinction values $k \leq 0.23$, the visibility windows of Mercury and Jupiter start overlapping; for values $k \geq 0.26$, Jupiter is still invisible on the $22 \mathrm{nd}$.
} 
around 5:40 $\mathrm{h}$ and that of Jupiter around 5:55 h. In Babylonian units, this difference of 15 min equals about 4 US (time degrees measured along the equator), roughly equivalent to a longitude difference of about 2 cubits (measured along the ecliptic).

We next turn to the planetary observations around the 28th day of month XII on the obverse of BM 32562. The first four lines of the text refer to observations on a date after the 26th day of the month and before the 30th day as follows:

BM 32562, 'Obv.

$\left.\# 1^{\prime} \quad \ldots . . . ..\right]$

$\# 2^{\prime} \quad 1 / 2$ cubit $[\ldots .$.$] ; all ni[ght....] first appearance [\ldots .$.$] it stood [\ldots$. behind....]

\#3' to the east; it was bright (and) high, [time interval (to sunrise/sunset)]....,

\#4' (ideal) first appearance on the 26th. The 30th, Saturn's first appearance in.... [....]

We discuss here the astronomical events in the early morning of the 29th day of month XII for an atmospheric extinction of $k=0.27$. The choice of this date is arbitrary. We could just as well have taken the 27 th or the 28 th day because the text indicates that it must have been between the 26th and the 30th day of the month. We describe the appearance and disappearance of planets visible on this day between 5:00 and 6:00 h:

- At 5:00 h, Venus is the only planet visible near the eastern horizon at an altitude of $3.8^{\circ}$.

- At 5:21 h, Mercury became visible at an altitude of $2.9^{\circ}$ with visual magnitude ${ }^{6}$ $V=4.8$. This is not its first appearance which occurred on day 21 , one week earlier.

- At 5:21 h, simultaneously with the appearance of Mercury, Saturn had its first appearance at $3.5^{\circ}$ above the horizon with magnitude $V=4.8$, after having been invisible for about 50 days. This is presumably the first appearance reported in the text.

- At 5:23 h, Jupiter became visible at $1.2^{\circ}$ above the horizon with magnitude $V=4.5$. Jupiter experienced its first appearance on day 23 so that it had already been visible during the six previous nights, shortly before sunrise.

- At 5:38 h, Saturn disappeared at an altitude of $6.8^{\circ}$ with magnitude $V=3.3$ in the brightening morning sky, having been visible for $17 \mathrm{~min}$.

- At 5:42 h, Mercury disappeared at an altitude of $7.0^{\circ}$ with magnitude $V=2.7$ in the brightening morning sky, having been visible for $21 \mathrm{~min}$.

- At 6:00 h, Venus and Jupiter are still visible.

\footnotetext{
6 The modern astronomical magnitude scale was defined in the nineteenth century in such a way that it stayed close to the original magnitude scale used by Ptolemy in the Almagest (second century AD) where stars were assigned magnitudes on a scale of one to six with stars of the 1st magnitude as the brightest and of the 6th magnitude as the faintest stars. So the smaller the magnitude the brighter the star. On the modern magnitude scale, the brightest stars and planets can even have negative magnitudes. Venus, by far the brightest object in the sky, has a visual magnitude of about -4 , and the brightest star Sirius has a visual magnitude of -1.2 .
} 


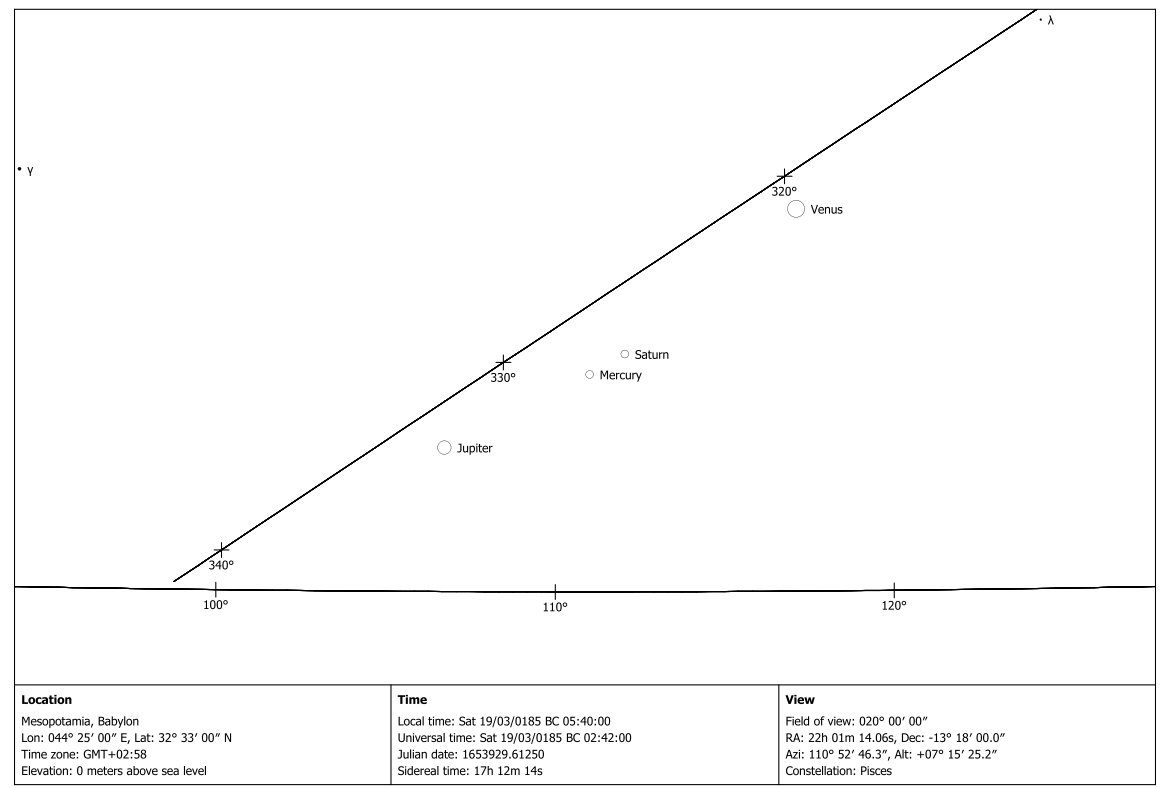

Fig. 2 The eastern morning sky in Babylon on day 29 of month XII in SE 126 (19 March 185 BC) at 5:40 h Local Time. The planets Jupiter, Mars, Mercury and Venus are visible. The sizes of the circles representing the planets are a measure of their relative brightness. The horizon and the ecliptic are indicated with azimuth and ecliptic longitude marked at $10^{\circ}$ intervals. [Produced with Cybersky 5.1.2; copyright (C) 2018 Stephen M. Schimpf]

The situation is illustrated in Fig. 2 where we show the eastern morning sky in Babylon on 19 March $185 \mathrm{BC}$ at 5:30 h. Notice that Mars is still invisible on this day.

Apparently, the observer thought that the planet that experienced its first appearance on this day was Mars and not Saturn because the first appearance of Saturn is reported 1 day later on day 30 close to Mars. Moreover, it seems that he not only confused Mars and Saturn but also Mercury and Mars (Saturn!). This is suggested by the statement that Mars was "bright and high", consistent with the fact that Mercury reached a maximum altitude of $7.0^{\circ}$ with magnitude $V=2.7$ (about one degree higher and one magnitude brighter than expected for Mars or Saturn on the day of its first appearance) and consistent with the fact that the text suggests that Mars (Mercury!) at its first appearance stood behind Mercury (Saturn!) to the east. So it seems that the observer takes Mercury for Mars and Saturn for Mercury.

This confusion is understandable in view of the exceptional constellation of the three planets Mercury, Mars and Saturn at this time: (1) located within a few degrees of each other, (2) with similar visual magnitudes (0.8-1.2), and (3) with expected ("ideal") dates of first appearance within a few days of each other (11-20 March).

We next discuss the astronomical phenomena observed during the night of day 30 of month XII recorded on BM 32562. The last four lines of the text preserved on the obverse of BM 32562 contain the following observations: 
BM 32562, 'Obv.

$\# 4$ ' $\quad . .$. . The 30th, Saturn's first appearance in.... [....]

$\# 5^{\prime}$ it stood [....] in front of Mercury to the west, $1 / 2$ cubit below.... [...., ]

$\# 6^{\prime}$ it stood [....] behind Mars to the east.... [....]

$\# 7^{\prime}$ (ideal) first appearance on the 29th. That month, [....]

Observations during the night of day 30 of month XII were also recorded on BM 46051 , both as a regular observation, as well as in the summary section at the end of the month. The first three lines of the text preserved on the obverse of BM 46051 contain the original observation:

BM 46051, 'Obv.'

\#1' [.... ar]ound the 30th, Saturn's first appearance be[low Mars,]

\#2' [it stood 2 cubits in fro]nt of Jupiter to the west, 31/2 cubits behind Mercury to the east; rising of Saturn to sunrise: $16^{\circ}$;

\#3' (ideal) first appearance [on the xth]

The final preserved lines on the obverse of tablet BM 45601 contain the observational summary of the astronomical events on day 30 :

BM 46051, 'Obv.'

\#8' $\quad$....; around the 30th, Saturn's first appearance $1 / 2$ cubit below Mars, it stood 2 cubits

\#9' [in front of Jupiter to] the west, $3 \frac{1}{2} 2$ cubits behind Mercury to the east; rising of Saturn to sunrise: $16^{\circ}$ ?;

This summary report is identical to the original observational record in lines 1-3, but it contains in addition a more accurate specification of the position of Saturn with respect to Mars.

The differences between the observations on day 30 of month XII, recorded on BM 32562 and on BM 46051, are so pronounced that they must have been carried out by different observers. We will call them observer A of BM 32562 and observer B of BM 46051. Before discussing the two different observational reports, we first describe the astronomical events in the early morning of day 30 day of month XII based on computations carried out for an atmospheric extinction of $k=0.25$ in Babylon. For this value of the extinction, Mars experiences its first appearance on this day, remaining visible for about $12 \mathrm{~min}$. We list the appearance and disappearance of planets visible between 5:00 and 6:00 $\mathrm{h}$ below:

- At 5:00 h, Venus is the only planet visible at the eastern horizon with magnitude $V=-0.9$ at an altitude of $3.8^{\circ}$.

- At 5:15 h, Saturn became visible at an altitude of $3.0^{\circ}$ with visual magnitude $V=4.9$. This is not its first appearance which occurred on 1 day earlier (see above).

- At 5:18 h, Mercury became visible at an altitude of $2.5^{\circ}$ with visual magnitude $V=4.8$. 


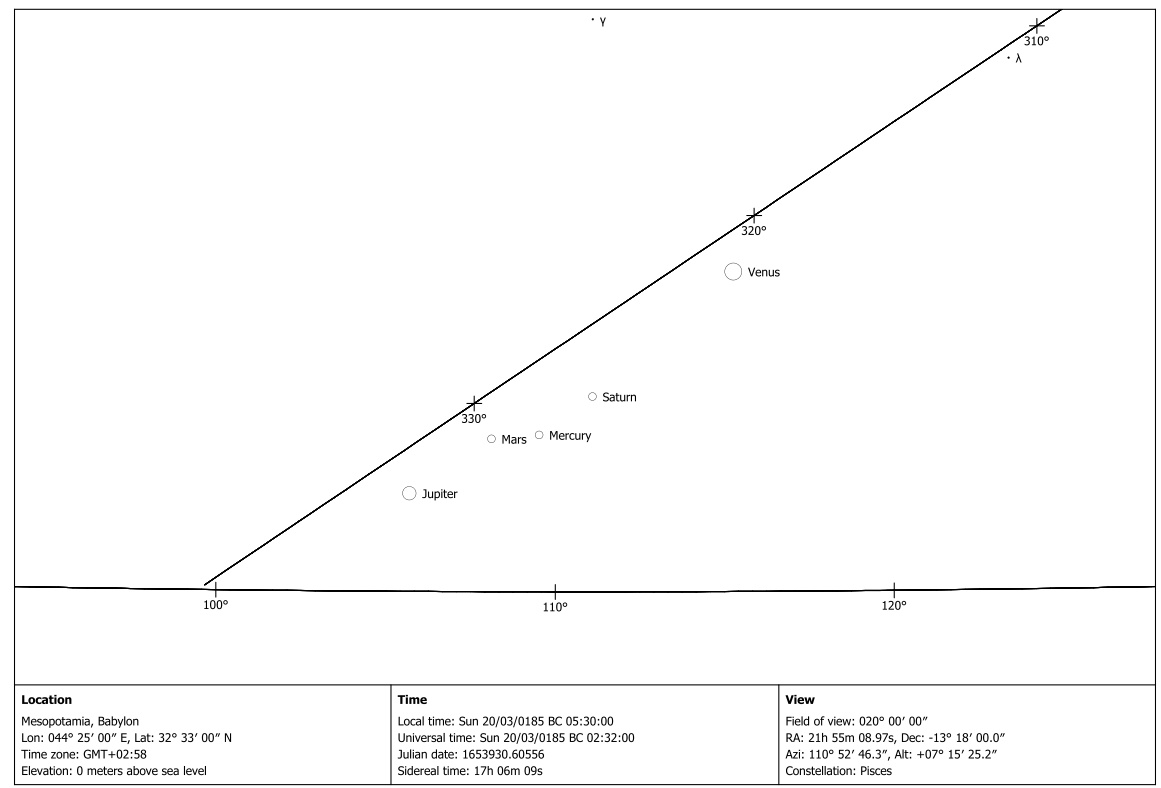

Fig. 3 The eastern morning sky in Babylon on day 30 of month XII in SE 126 (20 March 185 BC) at 5:30 h Local Time. All five planets are visible. The sizes of the circles representing the planets are a measure of their relative brightness. The horizon and the ecliptic are indicated with azimuth and ecliptic longitude marked at $10^{\circ}$ intervals. [Produced with Cybersky 5.1.2; copyright (C) 2018 Stephen M. Schimpf]

- At 5:19 h, Jupiter became visible at an altitude of $1.1^{\circ}$ with visual magnitude $V=4.5$.

- At 5:24 h, Mars had its first appearance, after having been invisible for about 200 days, at $3.5^{\circ}$ above the horizon with magnitude $V=4.6$. This is reported as the first appearance of Saturn in the text.

- At 5:35 h, Mars disappeared at an altitude of $5.7^{\circ}$ with magnitude $V=3.6$ in the brightening morning sky, having been visible for $12 \mathrm{~min}$.

- At 5:40 h, Saturn disappeared at an altitude of $7.9^{\circ}$ with magnitude $V=2.9$ in the brightening morning sky, having been visible for $26 \mathrm{~min}$.

- At 5:43 h, Mercury disappeared at an altitude of $7.3^{\circ}$ with magnitude $V=2.4$ in the brightening morning sky, having been visible for $26 \mathrm{~min}$.

- At 5:58 h, Jupiter disappeared at an altitude of $8.6^{\circ}$ with magnitude $V=-0.3$ in the brightening morning sky, having been visible for $40 \mathrm{~min}$.

- At 6:00 h, Venus is still visible at an altitude of $15.2^{\circ}$ with magnitude $V=-2.9$

The situation is illustrated in Fig. 3 where we show the eastern morning sky in Babylon on 20 March $185 \mathrm{BC}$ at 5:30 h when all five planets were visible.

From a comparison of the description of the relative positions of the planets Mercury, Mars and Saturn on day 30 in the text of BM 32562 with the actual situation in the morning sky on 20 March $185 \mathrm{BC}$ at 5:30 h in Fig. 3, it is clear that observer A is still unable to correctly identify the three planets. He takes Mars for Mercury, Mercury for Saturn and Saturn for Mars. The reddish colour characteristic of Mars 
is no help in the identification process because all three planets are reddened by the large extinction close to the horizon. With the above substitutions, the text makes perfect sense. From Fig. 3, we see that indeed Saturn (Mercury!) is in front of Mercury (Mars!) to the West, $1 / 2$ cubit $\left(0.7^{\circ}\right)$ below Mercury (Mars!), behind Mars (Saturn!) to the east.

We now turn to the report of the observations on day 30 of month XII by observer B on BM 46051. This observational record makes sense if we assume that he takes Mercury for Saturn, Saturn for Mars and Venus for Mercury. We find that Saturn (Mercury!) is slightly $\left(0.7^{\circ}\right)$ below Mars (Saturn!), $4.2^{\circ}$ in front of Jupiter and $7.5^{\circ}$ behind Mercury (Venus!). The mix up of Mercury, Saturn and Mars is quite understandable because the three planets are close together and all have about the same magnitude. Like his colleague A, observer B is similarly confused in taking Mercury for Saturn and Saturn for Mars. However, the fact that he takes Venus for Mercury is difficult to understand since Venus is about sixty times brighter than Mercury. This is most easily explained as a scribal error in an overall confusing situation.

We finally discuss the observations on the reverse sides of both tablets BM 32562 and BM 46051. The first three lines on the reverse side of BM 32562 read as follows:

BM 32562, Rev.'

\#1 [Night] of the 5 th, the moon was [....] above [....]

\#2 2? cubits, the moon [having passed] a little to the east [....]

\#3 Venus? was 8 fingers above Mars; last part of the night,.... [....],

\#4 [.... it was se]t? toward its inside [....]

and the first two lines of the preserved text on the reverse of BM 46051 read:

BM 46051, 'Rev.'

$\# 1^{\prime}$ [....] having passed [....] to the east. Night of the 5th, the m[oon was....] 8 fingers below? $\gamma$ Geminorum;

\#2' last part of the night, Mars was 3 fingers in front of Jupiter,

\#3' [.... The 5th, in the morning, very overcast, rain shower

The observation in line 1 of BM 46051 seems to be misread or inaccurate. In actual fact on the 5th day of month $\mathrm{XII}_{2}$ in the beginning of the night (24 March $185 \mathrm{BC}$ at 20:00 h), the moon was $6^{\circ}$ above and $0.6^{\circ}$ to the east of $\gamma$ Geminorum. The observation would have been correctly recorded if observer B would have written "behind" instead of "below". This observation was correctly recorded by observer A on BM 32562 in lines 1 and 2.

For our analysis of the remaining planetary observations on both tablets, we describe the astronomical events in the early morning of the 5 th day of month $\mathrm{XII}_{2}$ for an atmospheric extinction of $k=0.27$ :

- At 4:50 h, Venus is the only planet visible at the eastern horizon at an altitude of $2.3^{\circ}$.

- At 4:58 h, Saturn became visible at an altitude of $3.2^{\circ}$ with visual magnitude $V=5.0$. 


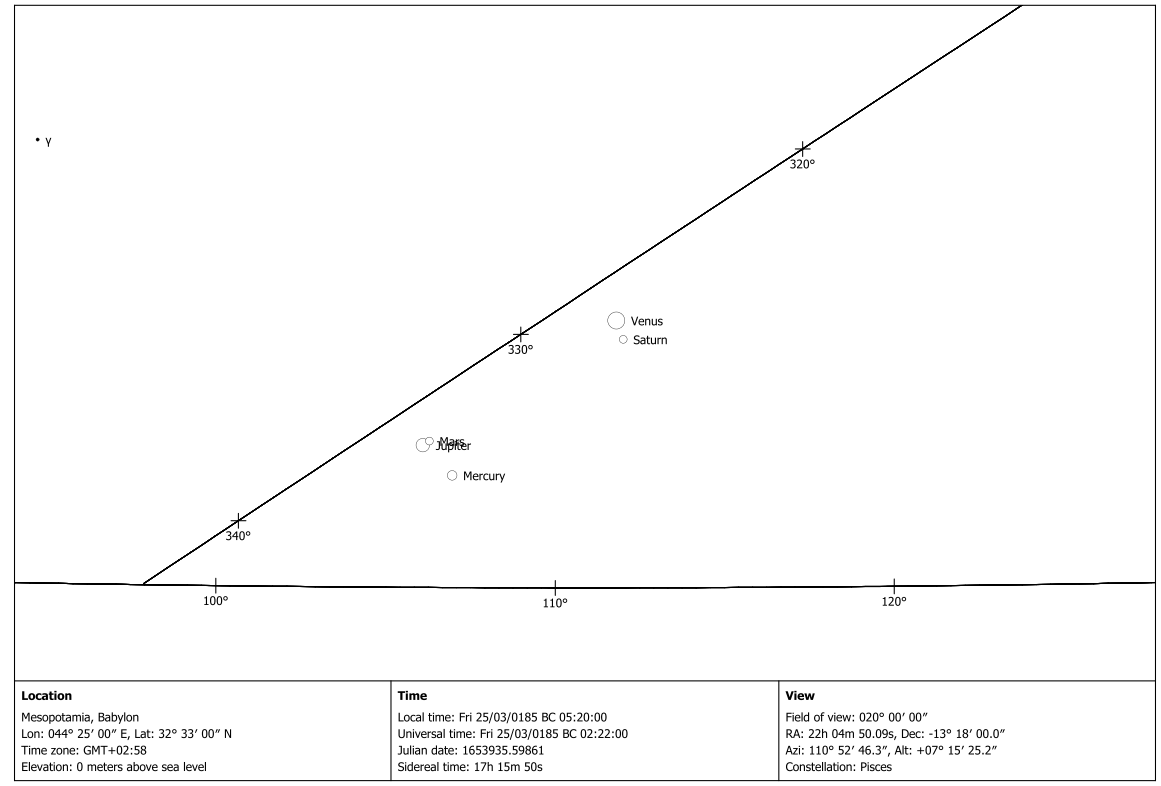

Fig. 4 The eastern morning sky in Babylon on day 5 of month $\mathrm{XII}_{2}$ in SE 126 (25 March 185 BC) at 5:20 h Local Time. All five planets are visible. The sizes of the circles representing the planets are a measure of their relative brightness. The horizon and the ecliptic are indicated with azimuth and ecliptic longitude marked at $10^{\circ}$ intervals. [Produced with Cybersky 5.1.2; copyright (C) 2018 Stephen M. Schimpf]

- At 5:03 h, Jupiter became visible at an altitude of $1.1^{\circ}$ with visual magnitude $V=4.9$.

- At 5:16 h, Mercury became visible at an altitude of $2.7^{\circ}$ with visual magnitude $V=4.7$.

- At 5:17 h, Mars became visible at $3.9^{\circ}$ above the horizon with magnitude $V=4.6$.

- At 5:27 h, Mars disappeared at an altitude of $5.9^{\circ}$ with magnitude $V=3.7$ in the brightening morning sky, having been visible for $11 \mathrm{~min}$.

- At 5:40 h, Saturn disappeared at an altitude of $7.9^{\circ}$ with magnitude $V=2.9$ in the brightening morning sky, having been visible for $26 \mathrm{~min}$.

- At 5:35 h, Mercury disappeared at an altitude of $6.4^{\circ}$ with magnitude $V=2.6$ in the brightening morning sky, having been visible for $20 \mathrm{~min}$.

- At 5:52 h, Jupiter disappeared at an altitude of $10.6^{\circ}$ with magnitude $V=-0.5$ in the brightening morning sky, having been visible for $50 \mathrm{~min}$.

- At 6:00 h, Venus is still visible at an altitude of $15.7^{\circ}$ with magnitude $V=-2.8$.

The situation is illustrated in Fig. 4 where we show the eastern morning sky in Babylon on 25 March $185 \mathrm{BC}$ at 5:20 h when all five planets were visible.

Observer A writes on BM 32562 in line 3 that on day 5 "Venus was 8 fingers above Mars". From a comparison with Fig. 4, it is clear that he remains consistent in confusing Mars and Saturn. Otherwise, the text is in excellent agreement with the actual situation in the late night sky of 25 March 185 BC: Venus is situated about $0.1^{\circ}$ to the west and about $0.6^{\circ}$ (6 fingers) above Mars (Saturn!). 
The observation in line 3 of BM 46051 is correctly recorded by observer $\mathrm{B}$. He is no longer confused about which planet is which because Fig. 4 shows that indeed on 25 March $185 \mathrm{BC}$ at 5:20 h Mars was $0.2^{\circ}$ (about 2 fingers) in front of Jupiter.

We struggled for some time trying to understand what astronomical phenomenon may have been described by the observation "last part of the night,... [... it was se]t? toward its inside [....]" in lines 3 and 4 of the reverse side of BM 32562. The term "it was set towards its inside" is consistently used in the Astronomical Diaries to refer to an upcoming conjunction of the Moon with a Normal Star, whereby the Moon covers the star (lunar occultation). ${ }^{7}$ However, during the night of the 5 th no lunar occultation of a Normal Star is foreseen.

As a solution of this dilemma, we propose that the technical term "it was set towards its inside" is used here to predict an expected "occultation" of the planet Saturn (Mars!) by Jupiter, or vice versa. Such an "occultation" could indeed be expected to occur soon because both planets have exactly the same ecliptic latitude and they are separated by only 8 fingers in ecliptic longitude according to observer $\mathrm{B}$ (of BM 46051). Indeed, $10 \mathrm{~h}$ later at 15:30 h during the day of the 5th an exceptionally close conjunction of Mars and Jupiter occurred when both planets were at an ecliptic longitude of $3.33^{\circ}$ Pisces with Mars and Jupiter at a distance of less than 1 arc minute from each other in ecliptic latitude $\left(1.15^{\circ}\right.$ and $1.14^{\circ}$, respectively $)$ which, if observed at night, would have led to both planets being seen as one object by a human observer.

We finally note that on this date, day 5 of month $\mathrm{XII}_{2}$ in SE 126 , the five planets were observed in Babylon to be most closely "massed" between Jupiter at $1^{\circ}$ Pisces and Venus at $8^{\circ}$ Pisces. $^{8}$ This unique planetary configuration was visible for about $10 \mathrm{~min}$ around 5:20 h on $25 \mathrm{March} 185 \mathrm{BC}$.

\section{Discussion}

The planetary configuration reported in BM 32562 and in BM 46051 is a unique and extremely rare astronomical event. It is known as "the massing of the planets". To determine how often a massing of the planets occurs, we searched for all dates between $600 \mathrm{BC}$ and $2000 \mathrm{AD}$ when each of the planets Mercury, Venus, Mars, Jupiter and Saturn was within $10^{\circ}$ of each of the other ones. This search resulted in the following five sets of dates: 22-28 March 185 BC, 27-29 November 47 BC, 3-6 October 332 AD, 21 June-3 July 710 AD and 16-20 September 1186 AD, so that a massing of the planets within $10^{\circ}$ happens on average about once every five hundred years.

During a massing of the planets, the Sun is always very close (within $27^{\circ}$ of Mercury) so that the planets will often not all be visible to the naked-eye observer.

\footnotetext{
7 Lunar occultations of stars are fairly rare phenomena. Between $261 \mathrm{BC}$ and $61 \mathrm{BC}$, the Astronomical Diaries (Sachs and Hunger 1989, 1996) contain 18 preserved records of occultations of Normal Stars by the Moon.

${ }^{8}$ Longitudes are given in the fixed Babylonian zodiac anchored to the Normal Star system (Huber 1958).
} 
Only when the Sun is far enough below the horizon while the planets are far enough above the horizon is there a chance that all planets can be seen. Otherwise, the twilight sky is too bright for some of the planets to become visible.

Both times that a massing of the planets occurred during the last six centuries BC, all five planets were simultaneously visible in Babylon under favourable weather conditions (no clouds and an atmospheric extinction less than 0.27 magnitudes per airmass): in $185 \mathrm{BC}$ from 20 to 30 March for about 10-40 min around 5:30 a.m. and in $47 \mathrm{BC}$ from 27 to 29 November for about 10-20 min around 6:00 a.m.

As we have seen above, there is an additional astronomical event which makes this planetary configuration even more unique: the exact conjunction of the planets Mars and Jupiter in the afternoon of 25 March 185 BC. Such a conjunction is also extremely rare. During the last six centuries BC, it happened three times: on 24 July $583 \mathrm{BC}$, on 25 March $185 \mathrm{BC}$ and on 13 July $156 \mathrm{BC}$. $^{9}$ The fact that this exact conjunction between Mars and Jupiter appears to have been correctly predicted by the Babylonian scholars is a remarkable achievement and a nice illustration of their astronomical craftsmanship.

The astronomical observations recorded in the two Astronomical Diaries, of which BM 32562 and BM 46051 are the remains, were obviously carried out by two different observers. There are a few other Diaries where it is clear that more than one observer must have been at work. Hunger and Pingree (1999, 143) mention the Diaries No. -171 and No. -163 as examples, but they also point out that of the twenty-four presently known multiple Diaries (19 duplicates, 3 triplicates and 2 quadruplicates) most are copies rather than reports by different observers.

The differences between the two Diaries studied here are in fact so pronounced that one may wonder whether the observations were not carried out at different observing sites. Support for this idea comes from the fact that the two observers sometimes confuse the planets in different ways which suggests little exchange of information between them. However, since there are no indications of the existence of Diary-type astronomical observing programs outside Babylon during the late Babylonian period (e.g. Steele 2016) and in view of the lack of evidence for the existence of Diaries from outside Babylon in the Esagila library and the Egibi archive (Clancier 2009; Pirngruber 2019), we are inclined to conclude that both observers were probably located in Babylon.

The present study illustrates several aspects of the visibility of stars and planets near the horizon during twilight that are often not realized or neglected by students of Babylonian astronomy. First, during twilight stars and planets never become visible exactly at the horizon but appear one or several degrees above the horizon, and secondly, planets, which do become visible near the horizon during twilight, may not be visible simultaneously but sequentially, depending on their relative position and on their magnitude.

\footnotetext{
${ }^{9}$ It is a lucky coincidence that a record of the observation of the exact conjunction of Mars and Jupiter on 13 July 156 BC has been preserved. In line 6 on the obverse of BM 45768 (Diary No. -155, Sachs and Hunger 1996), we read that in month IV of SE 156 during the first part of the night of the 19th "Mars came close to Jupiter, they turned into one star".
} 
Finally, our study clearly exposes one of the limitations of Babylonian nakedeye astronomy. When first appearances of the planets Mercury, Mars and Saturn are expected around the same date, it is nearly impossible to correctly identify them because their expected positions are only approximately known (with an accuracy of order $1^{\circ}$ ) while they have about the same visual magnitude (about 1.0) so that they become visible at about the same altitude above the horizon. ${ }^{10}$ In our days, this ambiguity could easily be resolved by telescopic observation, but in antiquity there was no way to identify the planets by visual means. The planets Venus and Jupiter are not subject to this kind of uncertainty at their first appearance because they can easily be identified based on their brightness: Jupiter is about 10 times $(\sim 2.5$ magnitudes) brighter and Venus even about 100 times ( $\sim 5$ magnitudes) brighter than Mercury, Mars and Saturn.

Acknowledgements We thank Alexander Jones for a critical reading of the manuscript and several useful suggestions for its improvement.

\section{Compliance with ethical standards}

Conflict of interest On behalf of all authors, the corresponding author states that there is no conflict of interest.

Open Access This article is licensed under a Creative Commons Attribution 4.0 International License, which permits use, sharing, adaptation, distribution and reproduction in any medium or format, as long as you give appropriate credit to the original author(s) and the source, provide a link to the Creative Commons licence, and indicate if changes were made. The images or other third party material in this article are included in the article's Creative Commons licence, unless indicated otherwise in a credit line to the material. If material is not included in the article's Creative Commons licence and your intended use is not permitted by statutory regulation or exceeds the permitted use, you will need to obtain permission directly from the copyright holder. To view a copy of this licence, visit http://creativecommons.org/licen ses/by/4.0/.

\section{References}

Britton, J.P. 2010. Studies in Babylonian Lunar Theory: Part III. The Introduction of the Uniform Zodiac. Archive for the History of Exact Sciences 64: 617-663.

Clancier, P. 2009. Les bibliothèques en Babylonie dans la deuxième moitié du Ier millènaire av. J.-C. Münster: Ugarit-Verlag.

de Jong, T. 2002. Early Babylonian Observations of Saturn: Astronomical Considerations. Alter Orient und Altes Testament 297: 175-192.

de Jong, T. 2007. Astronomical Dating of the Rising Star List in MUL.APIN. Wiener Zeitschrift für die Kunde des Morgenlandes 97: 107-120.

de Jong, T. 2012. Babylonian Observations of Venus: Arcus Visionis, Atmospheric Extinction and Observational Practice. Journal for the History of Astronomy 43: 391-409.

Huber, P. 1958. Über den Nullpunkt der babylonischen Ekliptik. Centaurus 5: 192-208.

Huber, P.J., A. Sachs, M. Stol, R.M. Whiting, E. Leichty, C.B.F. Walker, and G. van Driel. 1982. Astronomical Dating of Babylon I and Ur III. Malibu: Undena Publications.

\footnotetext{
${ }^{10}$ Or put differently, because they have about the same arcus visionis. See for instance Figure 1 of de Jong (2007) where the dependence of arcus visionis on magnitude is illustrated for the case of stars.
} 
Hunger, H. 2014. Astronomical Diaries and Related Texts from Babylonia, Vol. VII: Almanacs and Normal Star Almanacs. Vienna: Verlag der Österreichischen Akademie der Wissenschaften.

Hunger, H. 2021. Astronomical Diaries and Related Texts from Babylonia, vol. IV. Vienna: Verlag der Österreichischen Akademie der Wissenschaften.

Hunger, H., and D. Pingree. 1999. Astral Sciences in Mesopotamia. Leiden: Brill.

Langdon, S., J.K. Fotheringham, and C. Schoch. 1928. The Venus Tablets of Ammizaduga: A Solution of Babylonian Chronology by means of the Venus Observations of the first Dynasty. Oxford: Oxford University Press.

Parker, R.A., and W.H. Dubberstein. 1956. Babylonian Chronology 626 BC-AD 75. Providence: Brown University Press.

Pirngruber, R. 2019. The Museum Context of the Astronomical Diaries. In Keeping Watch in Babylon, ed. Johannes Haubold, John Steele, and Kathryn Stevens. Leiden: Brill.

Sachs, A.J., and H. Hunger. 1988. Astronomical Diaries and Related Texts from Babylonia, Vol. I: Diaries from 652 BC to 262 B.C. Vienna: Verlag der Österreichischen Akademie der Wissenschaften.

Sachs, A.J., and H. Hunger. 1989. Astronomical Diaries and Related Texts from Babylonia, Vol. II: Diaries from 261 BC to 165 B.C. Vienna: Verlag der Österreichischen Akademie der Wissenschaften.

Sachs, A.J., and H. Hunger. 1996. Astronomical Diaries and Related Texts from Babylonia, Vol. III: Diaries from 164 BC to 61 B.C. Vienna: Verlag der Österreichischen Akademie der Wissenschaften.

Steele, J.M. 2011. Goal-Year Periods and their Use in Predicting Planetary Phenomena. In The Empirical Dimension of Ancient Near Eastern Studies, ed. J. ed. Gebhard J. Selz and Klaus Wagensonner. Vienna: Lit Verlag.

Steele, J.M. 2016. The Circulation of Astronomical Knowledge between Babylon and Uruk. In The Circulation of Astronomical Knowledge in the Ancient World, ed. J.M. Steele. Leiden: Brill.

Publisher's Note Springer Nature remains neutral with regard to jurisdictional claims in published maps and institutional affiliations. 